\title{
ANÁLISE MULTIVARIADA DE ÍNDICES CLIMÁTICOS NA AMAZÔNIA OCIDENTAL
}

\author{
SANTOS, Daris Correia Dos - dariscorreia@gmail.com \\ Universidade Federal do Ceará
}

RESUMO: O objetivo deste trabalho foi estudar a dinâmica da variabilidade climática espacial e temporal da pluviosidade na Amazônia Ocidental, utilizando as técnicas multivariadas de Análise de Componentes Principais (ACP) e Análise de Agrupamento (AA). Foram utilizadas médias anuais da precipitação pluvial de quatro índices climáticos pluviais obtidas a partir de dados diários de 36 pontos de grade, extraídos da reanálise ERA-40 do ECMWF (European Centre for Medium-Range WeatherForecasts), para o período de 1970 a 2001. Na análise de componentes principais aplicada para os índices de precipitação pluvial PRCPTOT e SDII observaram-se que as cinco primeiras componentes apresentaram autovalores superiores ou igual a um e explicaram mais de $83 \%$ da variância total, para os demais índices, DCM foram retidas as nove primeiras componentes que explicaram $81,69 \%$ e para o DCS foram necessárias as quatro primeiras que explicaram $87,98 \%$. Com relação à determinação de áreas homogêneas verificou-se cinco regiões para PRCPTOT e SDII, quatro para DCS e seis para DCM. Conforme as análises da regionalização homogêneas usando CPs, conclui-se que o grande número de regiões obtidas reflete os sistemas de tempo produtores de precipitação na Amazônia Ocidental e a interação entre eles, bem como a interação deles com os efeitos locais.

Palavras-chave: Regiões homogêneas, Índices climáticos, Precipitação.

MULTIVARIATE ANALYSIS OF CLIMATIC INDICES IN THE WESTERN AMAZON

ABSTRACT: The aim of this work was to study the dynamics of spatial and temporal climate variability of rainfall in western Amazonia, using multivariate techniques of Principal Component Analysis (PCA) and Cluster Analysis (AA). Average annual rainfall of four stormwater climate indices derived from daily data from 36 grid points, extracted from the ERA- 40 reanalysis of the ECMWF (European Centre for Medium -Range Weather Forecasts) for the period 1970-2001 were used. In principal component analysis applied to the indices of rainfall and SDII PRCPTOT observed that the first five components had eigenvalues greater than or equal to one and explained over $83 \%$ of the total variance for the other indices, the nine DCM were retained first parts that explained $81.69 \%$ and DCS were necessary for the first four which explained $87.98 \%$. With respect to the determination of homogeneous areas there was five regions for PRCPTOT and SDII, four for DCS and six for DCM. As the analysis of homogeneous regionalization using CPs, it is concluded that the large number of regions obtained reflects the systems producing time of precipitation in western Amazonia and the interaction between them as well as their interaction with local effects.

Keywords: Homogeneous regions, climatic indices, rainfall.

\section{INTRODUÇÃO}

No estudo das variáveis climáticas sobre determinada área envolvendo vários pontos de observação, torna-se necessário o uso de determinadas técnicas estatísticas. Dentre estas, a mais utilizada, em várias áreas da ciência, é a análise multivariada, cujos principais objetivos são circunscrever a dimensão de uma matriz de dados, averiguar o comportamento espacial e temporal das variáveis consideradas e obter grupos homogêneos dessas variáveis.

Villar et al. (2009) estudaram a variabilidade espaço-temporal da precipitação nabacia amazônica através da Análise das Componentes Principais rotacionadas e diagnosticaram que as maiores variabilidades interdecenais e interanuais da chuva 
ocorrem no período chuvoso da região (dezembro a maio) e estão relacionadas a mudanças de longo prazo do Oceano Pacífico e eventos de ENOS, respectivamente.

Amanajás e Braga (2012) através da ACP analisaram os principais padrões climatológicos da precipitação na Amazônia Oriental e relacionaram com os principais mecanismos climáticos dos Oceanos Pacífico e Atlântico Tropical. Através dos resultados obtidos pela ACP foram detectados três padrões pluviométricos bem definidos que explicaram aproximadamente $92 \%$ da variância total dos dados; o padrão dominante definiu áreas de chuva que são influenciadas pela atuação da Zona de Convergência Intertropical (ZCIT), o segundo padrão espacial está associado a eventos de mesoescala, tal como as linhas de estabilização que favorecem as chuvas na região nos meses de maio a agosto e o terceiro padrão espacial evidencia a influência da atuação da Zona de Convergência do Atlântico Sul (ZCAS), sistema atuante no final da primavera e início do verão, bem como sistemas de escala local.

O conhecimento e a compreensão das alterações climáticas e dos seus efeitos são relevantes para formular cenários referentes ao comportamento futuro de variáveis relacionadas com o clima. Portanto, a presente pesquisa tem o objetivo calcular as componentes principais dos índices de precipitação relacionando-as com os sistemas de tempo e clima atuantes na Região e determinar as regiões homogêneas dos índices de extremo climático oriundos da precipitação da Amazônia Ocidental e relacionar com os eventos de tempo e clima atuantes na Região.

\section{MATERIAL E MÉTODOS}

O conjunto de dados de precipitação diária utilizado nesta pesquisa é proveniente de reanálise disponibilizados em pontos de grade com resolução espacial de 2,5 x 2,50 de latitude/longitude, para o período de 1970 a 2001, permitindo análises da configuração espacial da climatologia de cada índice de extremo climático estimado para a Amazônia Ocidental.

\subsection{Descrição geral da área de estudo}

A Amazônia Ocidental está situada entre as latitudes $13^{\circ} 42^{\prime} \mathrm{S}$ a $5^{\circ} 42^{\prime} \mathrm{N}$ e as longitudes $73^{\circ} 59^{\prime} \mathrm{W}$ a $56^{\circ} 20^{\prime} \mathrm{W}$, abrangendo os estados Amazonas, Acre, Rondônia e Roraima, localiza-se no centro geográfico da Amazônia Continental (Amazônia sul americana),ocupando uma área de $2.196 .743 \mathrm{~km}^{2}$. Essa área corresponde a 25,7\% do território brasileiro. Tem 6.409.000 habitantes, segundo estimativas do IBGE de 2012, foi estabelecida pelo $\S 4^{\circ}$ Art. $1^{\circ}$ do Decreto Lei no 291, de 28 de fevereiro de 1967. A distribuição sazonal da precipitação na Amazônia apresenta diferenças marcantes, principalmente, entre o norte e o sul, área que sofre influência constante da topografia, Figura 1. A precipitação média é da ordem de $2200 \mathrm{~mm}$.ano ${ }^{-1}$ (ESPINOZA et al., 2009). 


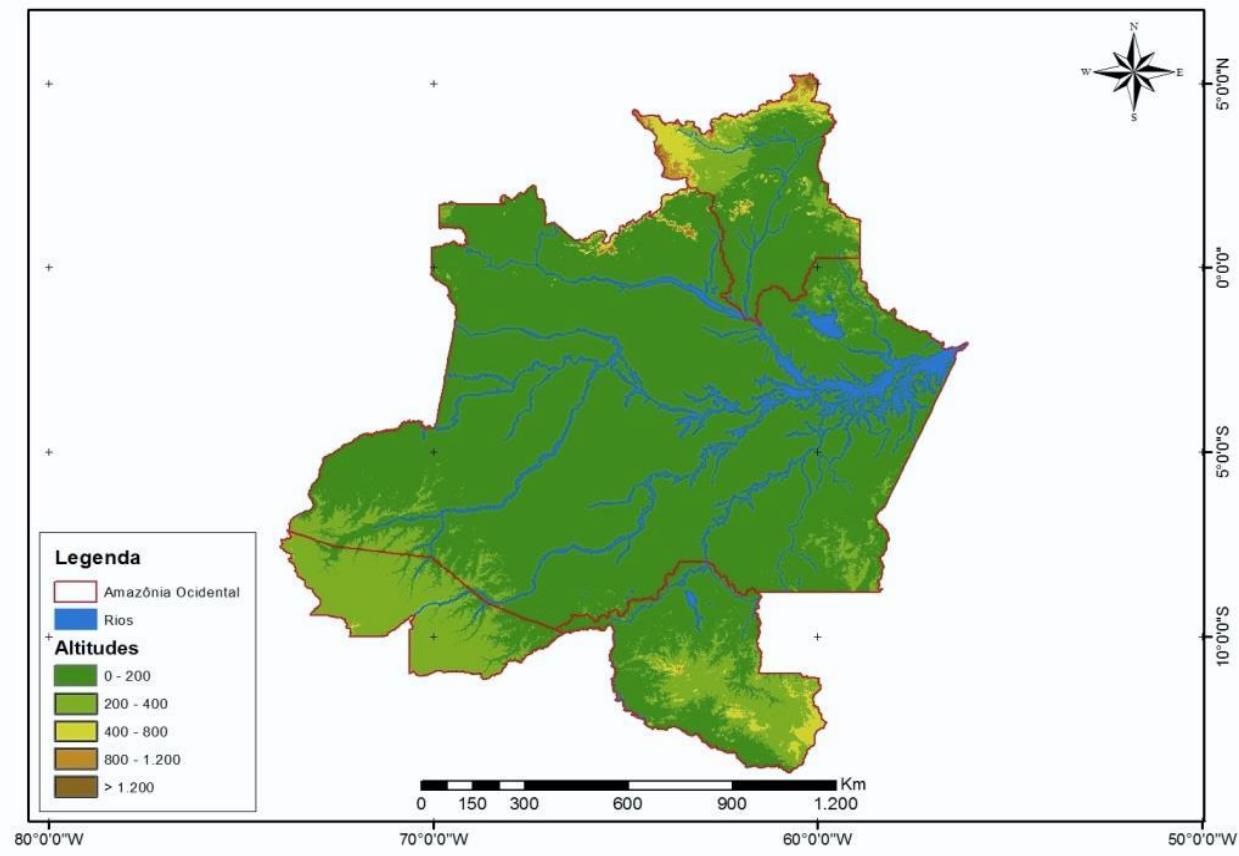

Figura 1- Relevo da Amazônia Ocidental.

\subsection{Dados climatológicos}

O conjunto de dados de precipitação para a Amazônia Ocidental utilizado neste trabalho é proveniente da reanálise ERA-40 do ECMWF (European Centre for MediumRange WeatherForecasts). Usando-se dados de 36 pontos de grade da reanálise de precipitação foram estimados os índices baseados na precipitação pluvial diária (1) precipitação total anual (PRCPTOT); (2) Índice simples de intensidade de precipitação diária (SDII); (3) dias consecutivos secos (DCS) e (4) dias consecutivos molhados (DCM). Estes índices foram estimados com o RClimDex. As analises estatísticas foram realizadas nos softwaresRClimDex, Excel e StatisticalPackage for the Social SciencesSPSS. E o Surfer foi utilizado na elaboração dos mapas.

$\mathrm{Na}$ Tabela 1 encontram-se os índices climáticos de precipitação definidos pelo ETCCDMI (Expert Team Change Detection Monitoring and Indices).

Tabela 1- Índices climáticos dependentes da precipitação pluvial diária, com suas definições e unidades. O RR é o valor da precipitação diária. $R R>1 \mathrm{~mm}$ representa um dia úmido e $\mathrm{RR}<1 \mathrm{~mm}$, um dia seco.

\begin{tabular}{|c|c|c|c|}
\hline Índices & Nome do Indicador & Definição & Unidade \\
\hline DCS & Dias consecutivos secos & $\begin{array}{l}\text { Número máximo de dias consecutivos com } \\
\mathrm{RR}<1 \mathrm{~mm}\end{array}$ & Dias \\
\hline DCM & Dias consecutivos molhados & $\begin{array}{l}\text { Número máximo de dias consecutivos com } \\
\text { RR }>1 \mathrm{~mm}\end{array}$ & Dias \\
\hline SDII & Índice simples de intensidadediária & $\begin{array}{l}\text { Média anual da precipitação quando } \\
P R C P \geq 1,0 \mathrm{~mm}\end{array}$ & $\mathrm{~mm}$ \\
\hline $\begin{array}{l}\text { PRCPTO } \\
\mathrm{T}\end{array}$ & $\begin{array}{l}\text { Precipitação total anual nos dias } \\
\text { úmidos }\end{array}$ & $\begin{array}{l}\text { Precipitação total anual nos dias úmidos } \\
(R R>1 \mathrm{~mm})\end{array}$ & $\mathrm{mm}$ \\
\hline
\end{tabular}




\subsection{Métodos}

A Análise de Componentes Principais rotacionadas aplicada aos dados médios de precipitação na Amazônia Ocidental produzem padrões espaciais associados a cada fator comum (pesos). A ACP fornece resultados a respeito da localização espacial dos principais modos de variabilidade e também a variação temporal desses modos. Os fatores rotacionados representam melhor a variabilidade dos dados que os não rotacionados, pois a variância explicada por cada nova variável é mais homogênea. E a análise de agrupamento permite identificar regiões homogêneas de acordo com as configurações de cada índice climático.

\subsubsection{Análise de Componentes Principais- ACP}

A análise de componentes principais é fortemente ligada à análise fatorial comum. Estas análises muitas vezes são usadas como sinônimas, mas não o são, mesmo existindo grandes semelhanças em seus métodos e resultados, pois ambas são análises fatoriais, que tem como objetivo principal identificar fatores não diretamente observáveis, a partir da correlação entre conjuntos de variáveis mensuráveis (CORRAR et al., 2007). A ACP permite detectar padrões e descrever um conjunto de variáveis através da criação de um número menor de dimensão e explorar a associação entre as variáveis estudadas a partir da identificação de fatores comuns. Em geral, a primeira solução obtida com a aplicação da ACP não fornece fatores que tenham a interpretação física mais adequada. Quando tal fato é observado para aumentar o poder explicativo dos fatores na análise, efetua-se o procedimento de rotação de fatores. Para realizar este processo o método mais utilizado é oVARIMAX (WILKS, 2006). Na escolha do número de fatores adequados ao estudo é utilizado o critério desenvolvido por Kaiser, (GARAYALDE et al, 1996). Na aplicação deste critério são excluídos os fatores com autovalores menores que um. As componentes principais são determinadas de forma que a primeira Componente Principal- $C P$, ou $C P(1)$ represente a maior parte da variabilidade total nos dados. Onde $\mathrm{CP}(1)$ é a combinação linear das variáveis observadas $X_{j} j=1,2, \ldots, p$ :

$$
C P_{(1)}=w_{(1)} x_{(1)}+w_{(1)} 2 x_{2}+\cdots+w_{(1) p} x_{p}(1)
$$

os pesos $\mathrm{w}_{(1) 1}, \mathrm{w}_{(1) 2}, \ldots, \mathrm{w}_{(1) \mathrm{p}}$ são determinados de forma a maximizar a relação entre a variação da $\mathrm{CP}_{(1)}$ com a variação total, sujeito a restrição

$$
\sum_{j}^{p}=1 w^{2}(1)=1(2)
$$

Assim, $\operatorname{Var}[C P(1)]$ é tão grande quanto possível sujeito a esta restrição sobre as constantes " $\mathrm{w}_{(i) j}$ ". A restrição é introduzida porque se isso não for feito, $\operatorname{Var}[C P(1)]$ pode ser aumentada fazendo simplesmente crescer qualquer um dos valores " $\mathrm{w}_{(i) j}$ ".

A CP(2), é a combinação linear ponderada das variáveis observadas que não foram correlacionadas na primeira combinação linear e que representa o montante máximo da variação total restante ainda não contabilizada pela $\mathrm{CP}(1)$.

$$
C P(2)=w(2) 1 X 1+w(2) 2 X 2+\ldots+w(2) p X p(3)
$$

onde, $\operatorname{Var}[C P(2)]$ é tão grande quanto possível sujeito as mesmas restrições impostas a $\mathrm{CP}(1)$ e que $\mathrm{CP}(1)$ e $\mathrm{CP}(2)$ possuam correlação igual a zero entre os seus dados. Os posteriores CP's são determinados da mesma forma, onde se existem " $m$ " séries de valores a $m$-ésima $\mathrm{CP}$ apresenta-se da seguinte forma:

$$
C P(m)=w(m) 1 X 1+w(m) 2 X 2+\ldots+w(m) p X p(4)
$$

A determinação $C P^{\prime}$ 's é realizada considerando a matriz de variáveis $X_{[p x m]}$, onde " $p$ " é o tamanho da série temporal, ou seja, o número de observações realizada ao longo do tempo, e " $m$ " é o número de séries, ou seja, de pontos espaciais que se deseja representar. 


$$
X=\left[\begin{array}{ccc}
x_{11} & \cdots & x_{1 p} \\
\vdots & \ddots & \vdots \\
x_{m 1} & \cdots & x_{m p}
\end{array}\right](5)
$$

A matriz de covariância de " $X$ " é simétrica e possui os elementos na diagonal principal iguais a $\operatorname{Var}[x 1]$ e o restante do termos são formados por $\operatorname{Cov}[x i x j]$. Se " $X$ " possui dados centrados em zero a matriz de covariância destes novos dados $R_{[p x p]}$ é formada pela matriz de correlação de " $X$ ".

$$
R=\left[\begin{array}{ccc}
1 & \cdots & r\left(x_{1} x_{p}\right) \\
\vdots & \ddots & \vdots \\
r\left(x_{p} x_{1}\right) & \cdots & 1
\end{array}\right](6)
$$

Os componentes principais são determinados resolvendo-se a equação característica da matriz: " $R$ ": $\operatorname{det}[R-\lambda /]=0$ ou $|R-\lambda /|=0$. Os resultados são " $p$ " raízes características chamadas de autovalores " $\lambda p$ " e para cada autovalor existe autovetor "wip":

$$
W=\left[\begin{array}{c}
w_{i 1} \\
w_{i 2} \\
\vdots \\
w_{i p}
\end{array}\right](7)
$$

A inversão da matriz de CP's para variáveis na escala padronizada "Y" pode ser realizada através.

$$
Y=C P x(W)-{ }^{1}(8)
$$

Como fisicamente cada componente está associada a um ou mais sistemas meteorológicos, analisou-se a representatividade dos sistemas climáticos /meteorológicos na variabilidade total de cada índice de extremo climático associando a configuração espaço-tempo das componentes com autovalor superior a 1,0.

\subsubsection{Métodos de Agrupamentos (Cluster)}

A análise de Clusters designa uma série de procedimentos estatísticos que podem ser usados para classificar variáveis por observação das semelhanças e dissemelhanças entre elas. A análise de clusters é organizada objetivando a formação de grupos relativamente homogêneos (Clusters) de determinada variável meteorológica.

O método de agrupamento pode ser descrito da seguinte forma: dado um conjunto de $\mathrm{n}$ indivíduos para os quais existe informação sobre a forma de $\mathrm{p}$ variáveis, o método agrupa os indivíduos em função da informação existente, de modo que os indivíduos de um grupo sejam tão semelhantes quanto possível e diferentes dos elementos dos grupos restantes.

Alguns algoritmos de análise de agrupamento operam com os dados organizados numa matriz de dados $n \times p$, conforme ilustrado na tabela abaixo:

$$
\left|\begin{array}{ccccc}
x_{11} & \ldots & x_{1 f} & \ldots & x_{1 p} \\
\vdots & \vdots & \vdots & \vdots & \vdots \\
x_{i l} & \ldots & x_{i f} & \ldots & x_{i p} \\
\vdots & \vdots & \vdots & \vdots & \vdots \\
x_{n 1} & \ldots & x_{n f} & & x_{n p}
\end{array}\right|
$$

Esta matriz representa os dados. Cada linha representa as coordenadas de um objeto i. Cada coluna representa os valores de um atributo assumidos por cada um dos $n$ objetos. 
Por outro lado, muitos algoritmos de agrupamento se aplicam em dados organizados numa matriz de dissimilaridade, onde o elemento da coluna $\mathrm{j}$ e linha $\mathrm{i}$ da matriz e o número $d(i ; j)$ representando a distância entre os objetos $i$ e $j$.

$$
\left|\begin{array}{ccccc}
0 & \ldots & & & \\
d(2,1) & 0 & & & \\
d(3,1) & d(3,2) & 0 & & \\
\vdots & \vdots & \vdots & \vdots & \vdots \\
d(n, 1) & d(n, 2) & d(n, 3) & \ldots & 0
\end{array}\right|
$$

\subsubsection{Distância euclidiana}

De acordo com Wilks (2006), a ideia central do agrupamento de um conjunto de dados distribuídos em pontos é o conceito de distância. A mais utilizada é a distância euclidiana em dados de vetores num espaço p-dimensional. Assim, a distância entre dois pontos $x_{i}$ e $x_{j}$ é:

$$
d i j=\left\|x_{i}-x_{j}\right\|=\left[\sum_{k=1}^{P}\left(x_{i, k}-x_{j, k}\right)^{2}\right]^{1 / 2}
$$

Quando se usam grandezas que não são diretamente comparáveis (com unidades e naturezas diferentes), a mudança de uma das unidades de medida pode alterar completamente o significado e o valor do coeficiente de semelhança. Essa é uma das razões pelas quais a padronização ou redução das variáveis se torna necessário. A redução é feita da seguinte forma: a matriz $\mathbf{X}_{(\mathrm{nxp})}$ é transformada numa outra $\mathbf{Z}_{(\mathrm{nxp})}$ tal que

$$
\begin{array}{r}
Z_{\mathrm{i}, \mathrm{k}}=\frac{\mathrm{x}_{\mathrm{ik}}-\bar{x}_{\mathrm{k}}}{\mathrm{S}_{\mathrm{k}}}(12 \mathrm{a}) \\
Z_{j, k}=\frac{x_{j k}-\bar{x}_{k}}{s_{k}}(12 \mathrm{~b})
\end{array}
$$

sendo $\bar{x}_{k}$ e $s_{k}$ a média e o desvio padrão dos valores na k-ésima coluna. Feita a transformação a distância euclidiana passa a ser:

$$
d i j=\left\|x z_{i}-z_{j}\right\|=\left[\sum_{k=1}^{P}\left(x_{i, k}-x_{j, k}\right)^{2}\right]^{1 / 2}(13
$$

ou, substituindo (12a) e (12b) em (13), temos:

$$
d i j=\left\|x_{i}-x_{j}\right\|=\left[\sum_{k=1}^{P} \frac{\left(x_{i, k}-x_{j, k}\right)^{2}}{S_{k}}\right]^{1 / 2}
$$

que é a média dos desvios quadráticos dividido pelos dados padronizados.

Tanto a distância euclidiana (medida de dissimilaridade) quanto o coeficiente de correlação (medida de similaridade) podem ser usados na determinação dos grupos. Para este fim existem dois tiposde métodos de agrupamento, os hierárquicos e os não hierárquicos. Dentre eles podem-se destacar os descritos por Wilks (2006), que são os seguintes: o método da ligação simples ou vizinho mais próximo, o da ligação completa ou vizinho mais distante, o método da centróide e o método de Ward.

\subsubsection{Método de Ward}

Nesta pesquisa optou-se usar o método de Ward, pois foi o que melhor representou a climatologia da Amazônia Ocidental. O método de Ward é um método de agrupamento de dados que forma grupos de maneira a atingir sempre o menor 
erro interno entre os vetores que compõe cada grupo e o vetor médio do grupo. Isto equivale a buscar o mínimo desvio padrão entre os dados de cada grupo. No método de Ward, os grupos de dados são formados em etapas. No início, têm-se $m$ grupos; ou seja, um grupo para cada vetor componente da base de dados. Neste estágio inicial o erro interno é nulo para todos os grupos, pois cada vetor que compõe cada grupo é o próprio vetor médio do grupo. Igualmente, o desvio padrão de cada grupo é nulo.

Na etapa subsequente, cada possibilidade de aglutinação entre os grupos 2 a 2 é verificada, e é escolhido o agrupamento que causa o menor aumento no erro interno do grupo. São $m \times m$ verificações. Desta forma, para uma base de dados com $\mathrm{m}$ valores observados, estas verificações exigem um grande esforço computacional caso o método seja executado em computador. Nota-se que a cada iteração tem-se $m_{i}$ grupos ( $i=$ número de iterações), no entanto, como o número de elementos pertencentes a cada grupo aumenta, é maior o número de cálculos para o erro interno de cada grupo. O agrupamento é feito diretamente através da equação:

$$
W=\sum_{i=1}^{n} x_{1}^{2}-\frac{1}{n\left(\sum x_{1}\right)}
$$

Outro método de encontrar regiões homogêneas para variáveis meteorológicas em determinada área geográfica é fazendo uso da análise de componentes principais. Para tanto plota-se os autovalores de cada CP em um gráfico, denominado descreeplot. Conforme método usado por Guedes et al., (2010) o objetivo é localizar um ponto que separa uma parte de forte inclinação para a esquerda, de outra parte com inclinação mais rasa para a direita; o número de componentes principais em que a separação ocorre é então considerado como o corte de truncamento, o critério inclinação screeplotnão envolve inferência estatística quantitativa (WILKS, 2006).

\section{RESULTADOS E DISCUSSÃO}

Por meio das análises de componentes principais dos índices de extremo climáticos realizou-se a análise de agrupamento para identificar regiões homogêneas de acordo com as características de cada variável, que neste caso, corresponderam às similaridades climáticas da precipitação e dos seus índices de extremos climáticos.

\subsection{Análise de componentes principais dos índices de precipitação}

A análise fatorial em componentes principais rotacionadas aplicada aos dados médios de precipitação e temperatura do ar na Amazônia Ocidental produziram padrões espaciais associados a cada fator comum (pesos). A ACP fornece resultados a respeito da localização espacial dos principais modos de variabilidade e também a variação temporal desses modos.

Para o índice de chuva PRCPTOT, observa-se na Tabela 2 que as cinco primeiras componentes apresentam fatores superiores a 1,0 e explicaram mais de $87 \%$ (rotacionado) da variância total. As configurações espaciais e temporais das cinco componentes são mostradas na Figura2. A Figura 2-a, primeira componente, mostra um máximo na parte centro-oeste do Estado do Amazonas, possivelmente decorrente da convecção local devido ao aquecimento diurno, enquanto, a Figura 2-b, segunda componente, mostra um gradiente meridional, provavelmente associado com a ZCIT. A Figura 2-c, terceira componente, mostra os maiores valores no extremo oeste do Acre e do Amazonas e no nordeste do Amazonas, que corresponde à topografia regional e umidade advectada pelos ventos alísios. A CP4 (Figura 2-d) apresenta uma configuração complicada, possivelmente representa a interação dos sistemas de 
tempo que atuam na Amazônia Ocidental. A CP5 explica 5,27\% da variância total e apresenta uma configuração com gradiente na direção sudoeste-nordeste (Figura 2e), este tipo de padrão deve estar associado à ZCAS e sistemas frontais austrais. A sexta CP foi excluída por não atender aos critérios de truncamento de componentes. Estas configurações diferem da precipitação média observada que apresentou a ZCIT com a responsável pela primeira componente e ZCAS/frentes frias austrais pela segunda. Isto ocorre porque as estações estão localizadas ao longo das margens dos rios e isto inibe configurações associada à topografia e de certa forma as convecções locais. No caso da reanálise estes fatores devem aparecer devido ao modelo utilizado na reanálise que contempla a topografia e a convecção cúmulo.
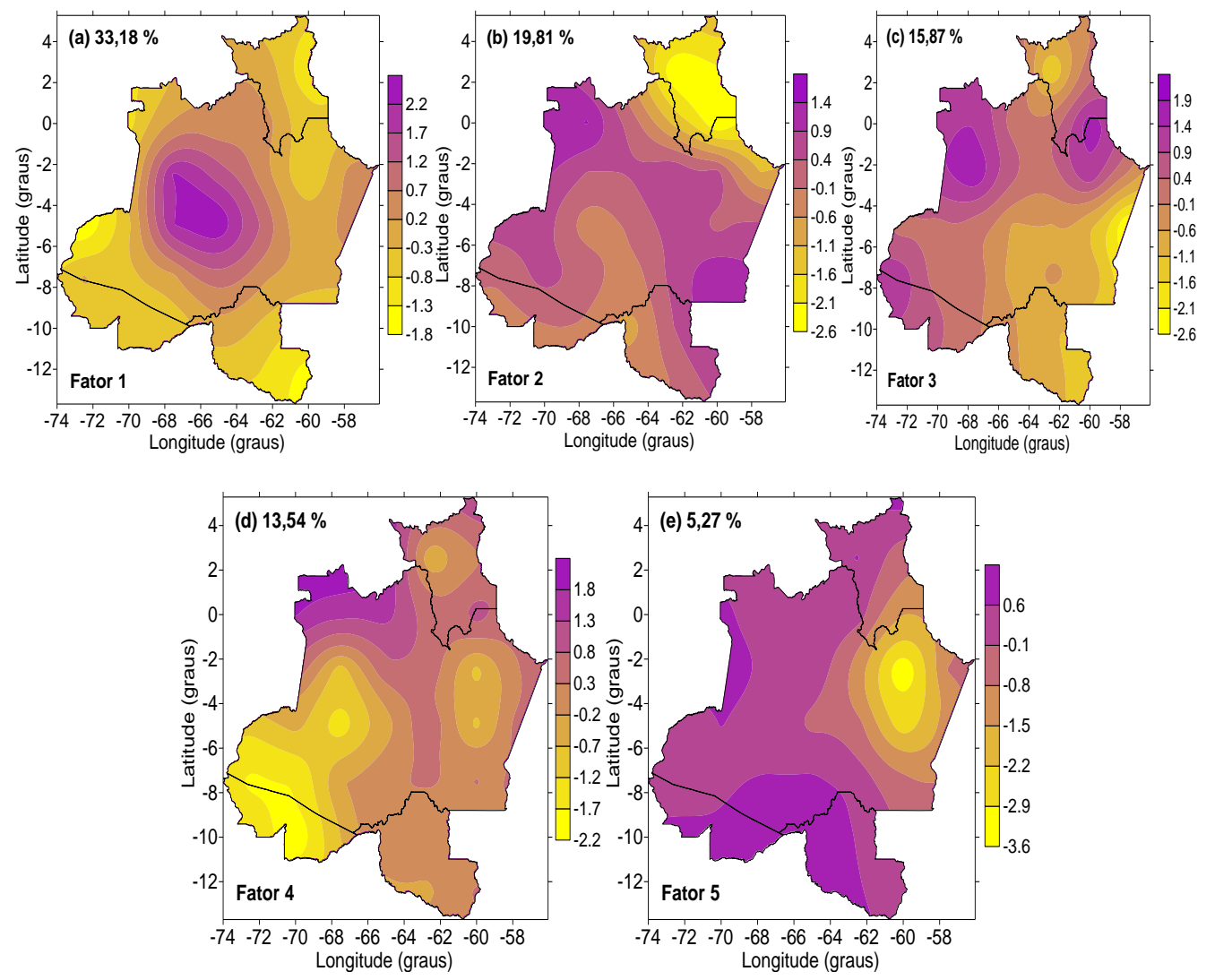

Figura 2- Distribuição espacial do modo de variabilidade do índice PRCPTOT, em mm/ano, para a Amazônia Ocidental, período de 1970 a 2001.

Tabela 2- Componentes e contribuição percentual da variância explicada do índice PRCPTOT para a Amazônia Ocidental, período de 1970 a 2001.

\begin{tabular}{cccccccc}
\hline & & & & Cargas & Rotacionadas \\
\hline & Total & Variância \% & Acumulado\% & Total & Total & Variância \% & Acumulado\% \\
\hline 1 & 18,08 & 56,51 & 56,51 & 18,08 & 10,62 & 33,18 & 33,18 \\
2 & 4,50 & 14,05 & 70,57 & 4,50 & 6,34 & 19,81 & 52,98 \\
3 & 2,34 & 7,32 & 77,89 & 2,34 & 5,08 & 15,87 & 68,85 \\
4 & 2,08 & 6,50 & 84,39 & 2,08 & 4,33 & 13,55 & 82,40 \\
5 & 1,05 & 3,28 & 87,67 & 1,05 & 1,69 & 5,27 & 87,67 \\
6 & 0,73 & 2,29 & 89,96 & & & $\ldots$ & $\ldots$ \\
$\ldots$ & $\ldots$ & $\ldots$ & $\ldots$ & $\ldots$ & $\ldots$ & & $\ldots$ \\
32 & 0,0003 & 0,0010 & 100 & & & & \\
\hline
\end{tabular}

As cinco primeiras componentes do índice SDII explicam 83,37\% da variância total (Tabela 3). Os campos das variabilidades espacial e temporal das cinco componentes são apresentados na Figura 3. Observa-se que as configurações são 
semelhantes as das PRCPTOT, Figura 2-a, 2-b, 2-c, 2-d e 2-e. As causas físicas destas configurações são as mesmas da PRCPTOT.
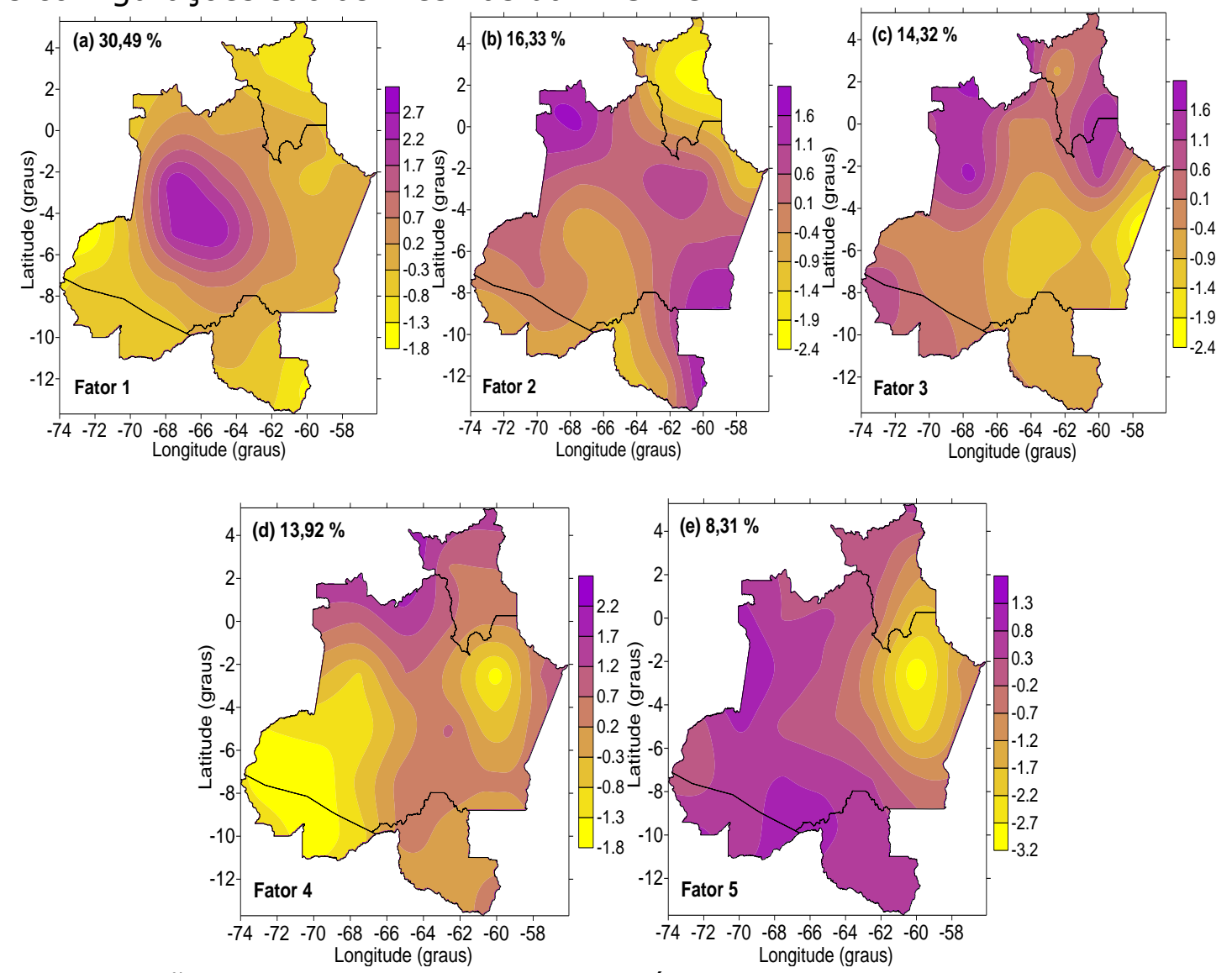

Figura 2-Distribuição espacial (mapa) e temporal (gráfico) das cinco CPs do SDII, em mm/dia, para Amazônia Ocidental, período de 1970 a 2001.

Tabela 3-Componentes e contribuição percentual da variância explicada do índice SDII para a Amazônia Ocidental, período de 1970 a 2001.

\begin{tabular}{|c|c|c|c|c|c|c|c|}
\hline & \multirow[b]{2}{*}{ Autovalor } & \multirow[b]{2}{*}{ Variância \% } & \multirow[b]{2}{*}{ Acumulado \% } & \multicolumn{4}{|c|}{ Cargas Rotacionadas } \\
\hline & & & & Total & Total & Variância \% & Acumulado \% \\
\hline 1 & 14,98 & 46,82 & 46,82 & 14,98 & 9,76 & 30,49 & 30,49 \\
\hline 2 & 5,12 & 16,00 & 62,81 & 5,12 & 5,22 & 16,33 & 46,82 \\
\hline 3 & 2,93 & 9,15 & 71,96 & 2,93 & 4,58 & 14,32 & 61,13 \\
\hline 4 & 2,42 & 7,56 & 79,52 & 2,42 & 4,46 & 13,92 & 75,06 \\
\hline 5 & 1,23 & 3,85 & 83,37 & 1,23 & 2,66 & 8,31 & 83,37 \\
\hline 6 & 0,83 & 2,59 & 85,96 & & & & \\
\hline$\ldots$ & $\ldots$ & $\ldots$ & $\ldots$ & $\ldots$ & $\ldots$ & $\ldots$ & $\ldots$ \\
\hline 32 & 0,000 & 0,001 & 100 & & & & \\
\hline
\end{tabular}

Para o índice DCM observou-se que as nove primeiras CPs apresentam autovalores maior que 1,0 e o conjunto destas nove CPsexplicam $81,69 \%$ da variância total (Tabela 4). Não é possível afirmar categoricamente que as cargas do índice DCM, Figura 4, representam fielmente os dias úmidos na área em estudo, haja vista que os valores das variâncias explicadas dessas CPs são baixos quando comparados à variância total explicada. A principal razão para a obtenção de nove CPs do índice DCM com score significativo é devido ao fato de que a precipitação na Amazônia Ocidental ocorre de forma convectiva. Como isto em um determinado dia 
pode ocorrer precipitação extremamente forte em uma localidade e na adjacente a precipitação seja mais amena. Em outro dia a precipitação mais forte já ocorre em outra localidade. Portanto, o total anual ou até mesmo mensal de precipitação entre as diversas localidades são próximos, entretanto, a variabilidade diária pode ser diferente.
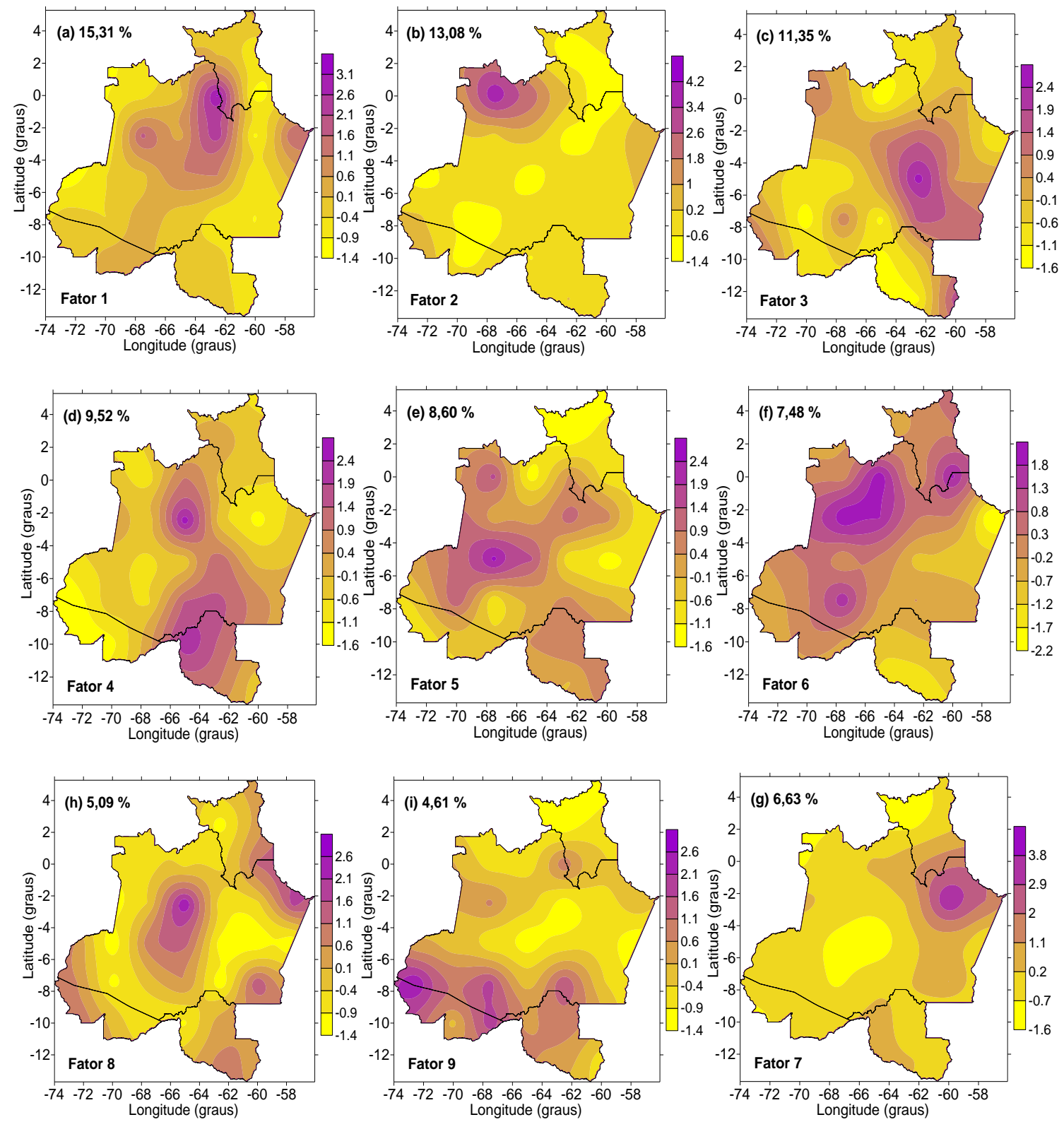

Figura 4- Distribuição espacial (mapa) e temporal (gráfico) do índice DCM para a Amazônia Ocidental, período de 1970 a 2001. 
Tabela 4. Componentes e contribuição percentual da variância explicada da média do índice DCM anual.

\begin{tabular}{cccccccc}
\hline \multicolumn{7}{c}{ Cargas } & Rotacionadas \\
& Autovalor & $\%$ & $\begin{array}{c}\text { Acumulado } \\
\%\end{array}$ & Total & Total & Variância \% & Acumulado \% \\
\hline 1 & 12,61 & 39,40 & 39,40 & 12,61 & 4,90 & 15,31 & 15,31 \\
2 & 2,49 & 7,78 & 47,19 & 2,49 & 4,19 & 13,08 & 28,39 \\
3 & 2,27 & 7,08 & 54,27 & 2,27 & 3,63 & 11,35 & 39,74 \\
4 & 2,11 & 6,58 & 60,85 & 2,11 & 3,05 & 9,53 & 49,27 \\
5 & 1,60 & 5,00 & 65,85 & 1,60 & 2,75 & 8,60 & 57,87 \\
6 & 1,49 & 4,66 & 70,51 & 1,49 & 2,39 & 7,48 & 65,36 \\
7 & 1,30 & 4,07 & 74,58 & 1,30 & 2,12 & 6,63 & 71,99 \\
8 & 1,26 & 3,94 & 78,52 & 1,26 & 1,63 & 5,10 & 77,09 \\
9 & 1,02 & 3,18 & 81,69 & 1,02 & 1,47 & 4,61 & 81,69 \\
10 & 0,88 & 2,76 & 84,45 & & & & $\ldots$ \\
$\ldots$ & $\ldots$ & $\ldots$ & $\ldots$ & $\ldots$ & $\ldots$ & & \\
32 & 0,00 & 0,01 & 100 & & & & \\
\hline
\end{tabular}

Para o índice DCS verificou-se que as quatro primeiras CPs apresentam autovalores superiores a 1,0 e explicam aproximadamente $88 \%$ da variância total (Tabela 5). As configurações espaciais e temporais das quatro CPs são apresentadas na Figura 5. Observa-se que a CP1 apresenta maiores valores na parte sudeste e a CP2 no norte (Figuras 5-a e 5-b). A distribuição espacial das cargas da CP3 e CP4, Figuras 5-c e 5-d mostram valores elevados de DCS na parte norte e sul, respectivamente.
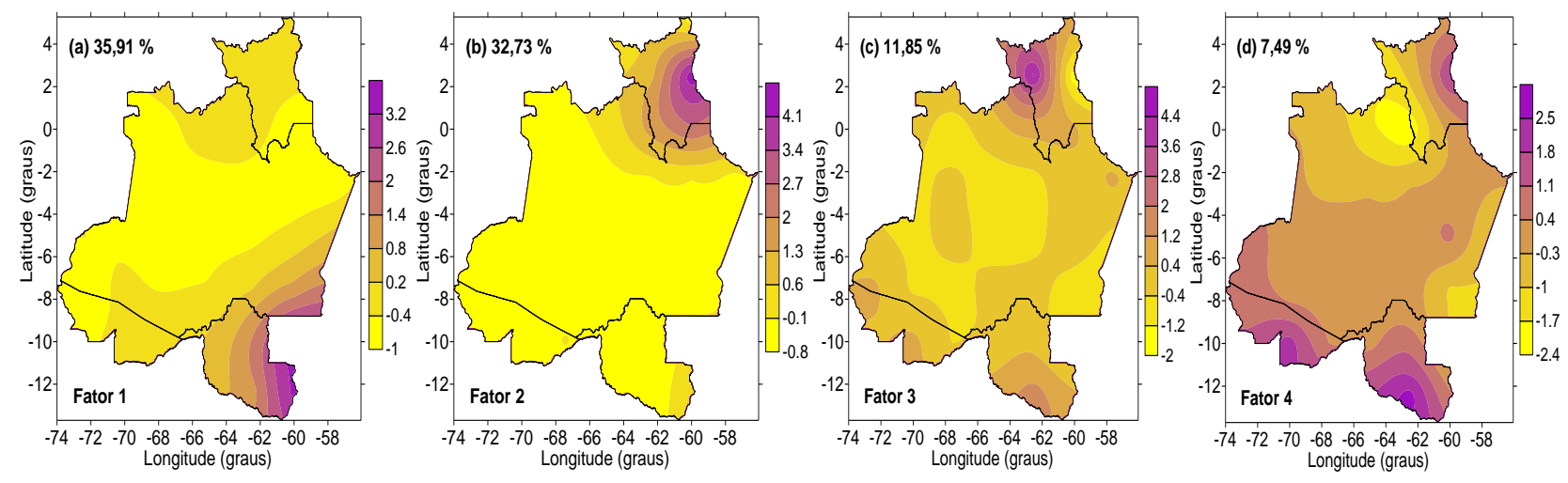

Figura 5- Distribuição espacial (mapa) e temporal (gráfico) do índice DCS para a Amazônia Ocidental, período de 1970 a 2001.

Tabela 5-Componentes e contribuição percentual da variância explicada do índice DCS para a Amazônia Ocidental, período de 1970 a 2001.

\begin{tabular}{|c|c|c|c|c|c|c|c|}
\hline & & & & & Cargas & Rotacionadas & \\
\hline & Autovalor & Variância \% & Acumulado \% & Total & Total & Variância \% & Acumulado \% \\
\hline 1 & 19,74 & 61,67 & 61,67 & 19,74 & 11,49 & 35,91 & 35,91 \\
\hline 2 & 5,60 & 17,49 & 79,16 & 5,60 & 10,47 & 32,73 & 68,64 \\
\hline 3 & 1,81 & 5,66 & 84,83 & 1,81 & 3,79 & 11,85 & 80,49 \\
\hline 4 & 1,01 & 3,15 & 87,98 & 1,01 & 2,40 & 7,49 & 87,98 \\
\hline 5 & 0,80 & 2,49 & 90,47 & & & & \\
\hline 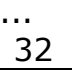 & 0,00 & 0,00 & 100,00 & $\ldots$ & $\ldots$ & $\ldots$ & $\ldots$ \\
\hline
\end{tabular}

\subsection{Caracterização e Análise das regiões homogêneas}

Após as análises da configuração espacial da climatologia anual da precipitação da Amazônia Ocidental e das análises de componentes principais dos índices de extremo climáticos realizou-se a análise de agrupamentos para identificar regiões 
homogêneas de acordo com as características de cada variável, que neste caso, corresponderam às similaridades climáticas da precipitação e dos seus índices de extremos climáticos.

A distribuição das regiões homogêneas da precipitação reflete sua associação com parâmetros meteorológicos em escala intra e intersazonais e interanual, fisiológicos (vegetação), topográficos, continentalidade e latitudinal explicando a localização de cada região e características climáticas existentes, o método de Ward foi o que melhor representou a natureza dos dados através da semelhança com as características climáticas, utilizando como medida de similaridade (ou função de agrupamento) a distância euclidiana.

O número escolhido de componentes principais foi baseado no critério de truncamento de Kaiser (KAISER, 1958), que considera como mais significativos os autovalores cujos valores sejam superiores à unidade (GARAYALDE et al., 1996). Efetuando-se uma pequena inspeção no screeplot, Figura 6, o gráfico apresenta tendência de paralelismo com o eixo das abscissas a partir do sexto fator, ou seja, sem influência. Observa-se uma seta indicando onde, a partir da qual, nos valores dos autovalores são inferiores a unidade e tornam-se quase constantes, ou seja, que a partir desse ponto em destaque a variância explicada por cada CP pode ser considera desprezível, daí a justificativa para a escolha de cinco regiões do índice SDII.
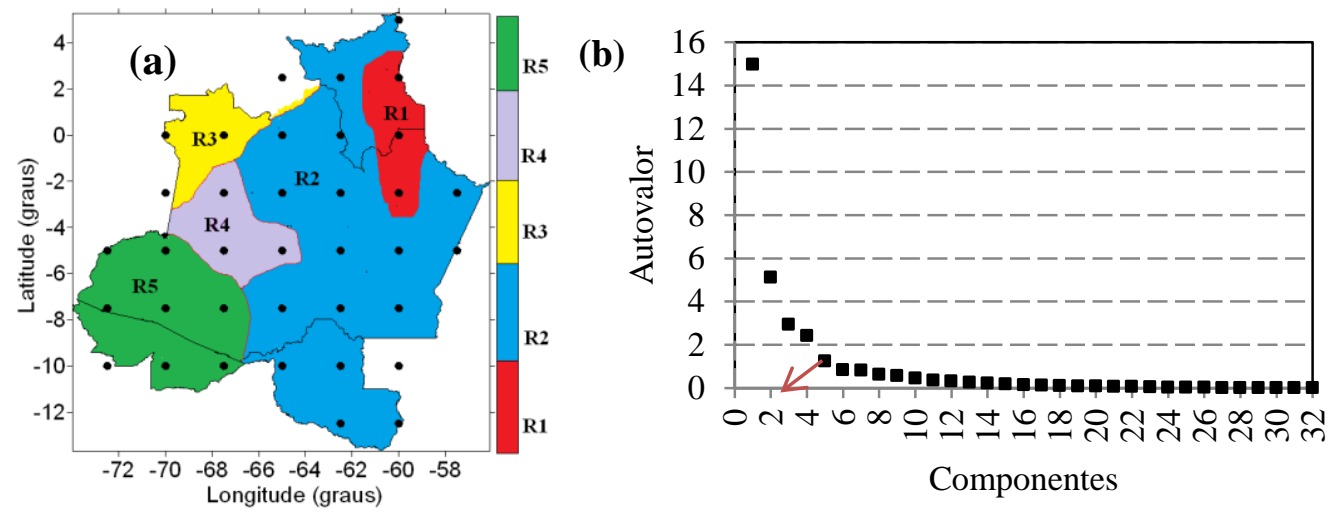

Figura 6-Regiões homogêneas da análise de CP do índice SDII (a) distribuição espacial e (b) estágios dos cálculos definindo o número de regiões homogêneas para a Amazônia Ocidental, período 1970-2001.

A Figura 7 apresenta as regiões homogêneas do índice DCS, para Amazônia Ocidental, obtidas por meio da análise de $\mathrm{CP}$, também mostra o gráfico com a tendência de paralelismo com o eixo das abscissas a partir do quinto fator, ou seja, a partir da quinta $\mathrm{CP}$ a influência das CPs na variância explicada é desprezível. Observa-se uma seta indicando onde, a partir da qual, os valores dos autovalores de cada CP tornam-se muito próximo de zero, indicando que a partir desse ponto as CPS devem ser desconsideradas para explicar a variância espacial e temporal do índice DCS, daí a justificativa para a escolha de quatro regiões homogênea do índice DCS pelo método da análise de componentes principais. 

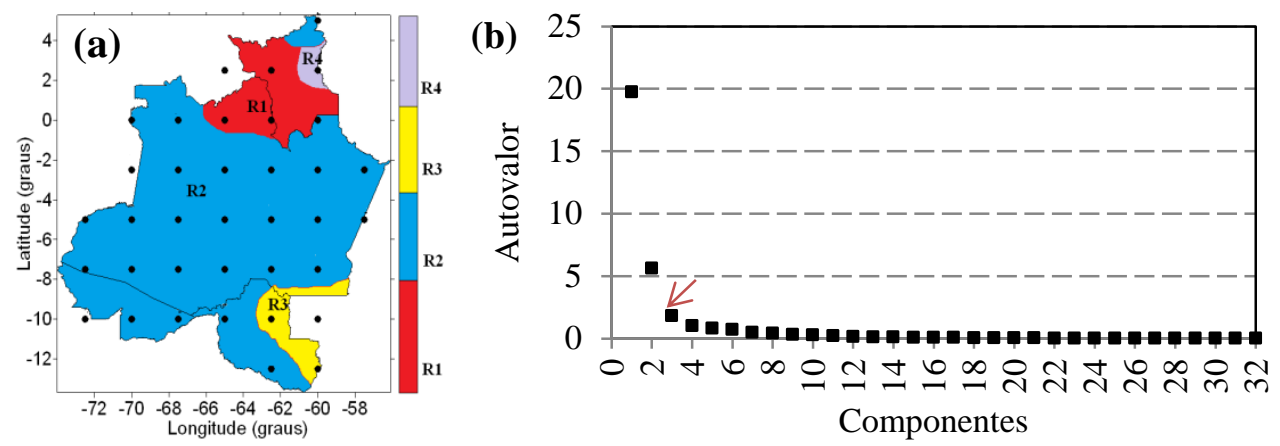

Figura 7-Regiões homogêneas da análise de CP do índice Dias Consecutivos Secos-DCS (a) distribuição espacial e estágios dos cálculos (b) definindo o número de regiões homogêneas para a Amazônia Ocidental, período 1970-2001.

A Figura 8apresenta a configuração espacial dos grupos homogêneo do índice $D C M$, pelo método da análise de CP e o gráfico com a tendência de paralelismo com o eixo das abscissas a partir do sétimo fator, ou seja, a partir da sétima CP a variância explicada por cada CPs é quase nula. Observa-se uma seta indicando que a partir desse ponto em destaque, as CPs devem ser desprezadas para agrupamento do conjunto de dados, daí a justificativa para a escolha de seis regiões do índice DCM.

Para este índice se faz necessário um número mais elevado de regiões homogêneas. Isto decorre do processo de precipitação na Amazônia, em geral, ser convectivo, fazendo com que ocorra chuva em uma área e não ocorra na área vizinha, no dia seguinte a configuração da precipitação modifica, ou seja, na área que chove, no dia anterior, volta a chover ou não, mas chove na área vizinha.
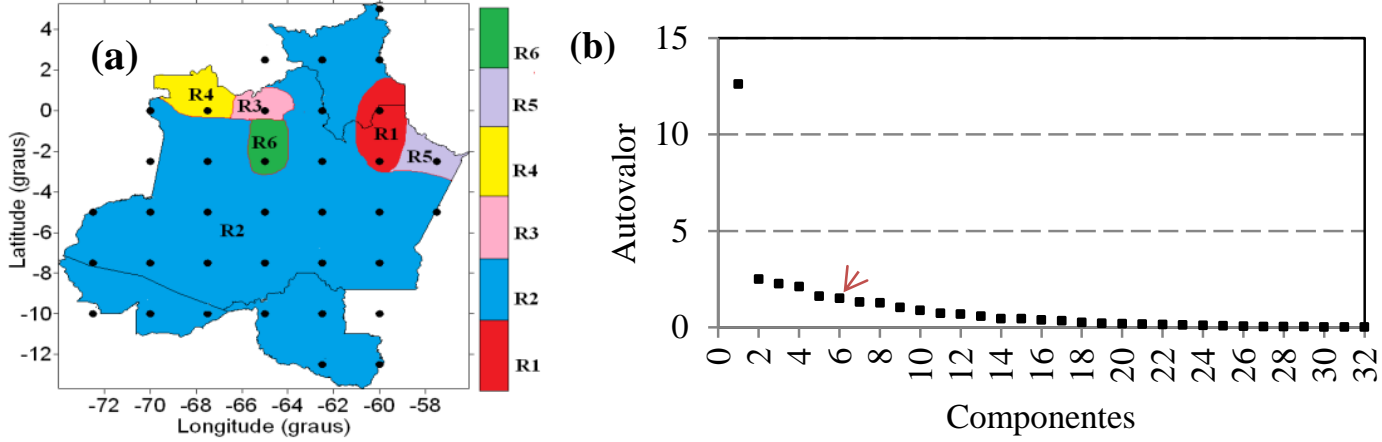

Figura 8-Regiões homogêneas do índice DCM (a) distribuição espacial e (b) Estágios dos cálculos definindo o número de regiões homogêneas para a Amazônia Ocidental, período 1970-2001.

A Figura 9 apresenta os valores dos autovalores de cada componente. Observa-se que a partir da sexta CP existe uma tendência de paralelismo entre os autovalores com o eixo das abscissas, ou seja, sem influência de cada CP na variância explicada do conjunto de dados do índice PRCPTOT. Observa-se uma seta indicando onde a partir desse ponto a influência das CPs torna-se desprezível, daí a justificativa para a escolha de cinco regiões do índice PRCPTOT, por meio da ACP para a Amazônia Ocidental. 

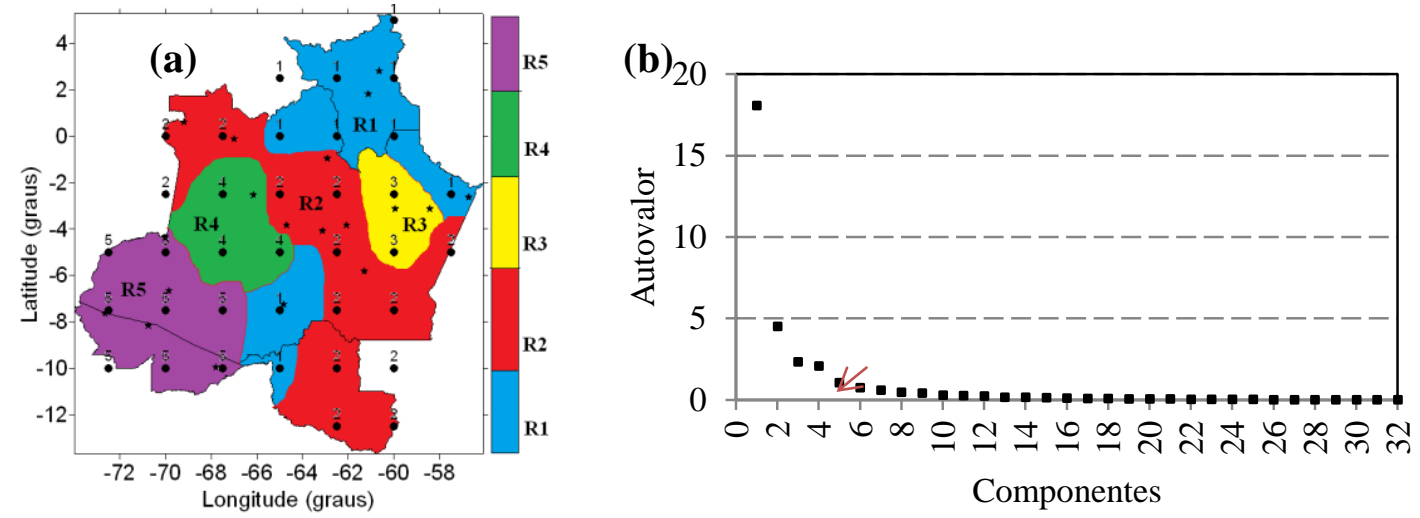

Figura 9-Regiões homogêneas do índice PRCPTOT pela análise de CP (a) distribuição espacial e (b) estágios dos cálculos definindo o número de regiões homogêneas para a Amazônia Ocidental, período 1970-2001.

\section{CONCLUSÕES}

A análise de componentes principais aplicada para os índices de precipitação pluvial PRCPTOT e SDII observou-se que as cinco primeiras componentes apresentaram autovalores superiores ou igual a um e explicaram mais de $83 \%$ da variância total, para os demais índices, DCM foram retidas as nove primeiras componentes que explicaram $81,69 \%$ e para o DCS foram necessária as quatro primeiras que explicaram $87,98 \%$.

Com relação à determinação de áreas homogêneas verificaram-se cinco regiões para PRCPTOT e SDII, quatro para DCS e seis para DCM. Conforme as análises da regionalização homogêneas usando $\mathrm{CPs}$, conclui-se que o grande número de regiões obtidas reflete os sistemas de tempo produtores de precipitação na Amazônia Ocidental e a interação entre eles, bem como a interação deles com os efeitos locais.

\section{REFERÊNCIAS}

AMANAJÁS, J. C.; BRAGA, C. C. Padrões espaço-temporal Pluviométricos na Amazônia Oriental utilizando Análise Multivariada. Revista Brasileira de Meteorologia, v.27, n.4, p. 323-338, 2012.

CORRAR, L. J.; PAULO, E.; FILHO, J. M. D. Análise Multivariada: para os cursos de administração, ciências contábeis e economia. Ed. Atlas. São Paulo, 2007.

ESPINOZA, J. C; RONCHAIL, J; GUYOT, J. L; FILIZOLA, N; NORIEGA, L; ORDONEZ, J. J; POMBOSA, R; ROMERO, H. Spatio-Temporal rainfallvariability in theAmazonBasin Countries (Brazil, Peru, Bolivia, ColombiaandEcuador). InternationalJournalofClimatology. v. 29, p. 1574 - 1594, 2009.

GARAYALDE, E. J. G.; SILVA, M.G.A. da; TAVARES, SÁ A. de. Classificação mesoclimática da região sul do Brasil pela análise de componentes principais. Anais... In:Congresso Interamericano de Meteorologia, 1., CongressoBrasileiro de Meteorologia, Brasília, 1996.

GUEDES, R. V. S; LIMA, F. J. L.; AMANAJÁS, J. C.;Célia Campos BRAGA, C. C. Análise em componentes principais da precipitação pluvial no estado do piauí e pelo método de ward.

Revista de Geografia. Recife: UFPE - DCG/NAPA, v. 27, n. 1, jan/mar. 2010

KAISER, H.F. The varimax criterion for analytic rotation in factor analysis.Psychometrika, v. 23, n, 3, p. 187-200, 1958. 
VILLAR, J. C. E.; RONCHAIL, J.; GUYOT, J. L.; COCHONNEAU, G.; NAZIANO, F.; LAVADO, W.; OLIVEIRA, E.; POMBOSA, R. VAUCHEL, P. Spatio-temporal rainfall variability in the Amazon basin countries (Brazil, Peru, Bolivia, Colombia, and Ecuador). International Journal of Climatology, v.29, n. 11, p. 1574-1594, 2009.

WILKS, D. S. Statistical Methods in the Atmospheric Sciences.2 ${ }^{\text {a }}$ Edition. California: Elsevier Science \& Technology Books. Academic Press, 2006. 